Research Article

\title{
Feature Extraction of Double Pulse Metal Inert Gas Welding Based on Broadband Mode Decomposition and Locality Preserving Projection
}

\author{
Yanfeng Peng $\mathbb{D},{ }^{1,2}$ Zucheng Wang, ${ }^{1,2}$ Kuanfang He $\mathbb{D}^{1,2,3}$ Yanfei Liu, ${ }^{4}$ Qingxian Li, ${ }^{5}$ \\ Liangjiang Liu, ${ }^{5}$ and Xianyu $\mathrm{Zhu}^{5}$ \\ ${ }^{1}$ Hunan Provincial Key Laboratory of Health Maintenance for Mechanical Equipment, \\ Hunan University of Science and Technology, Xiangtan 411201, China \\ ${ }^{2}$ Engineering Research Central of Advanced Mining Equipment, Ministry of Education, \\ Hunan University of Science and Technology, Xiangtan 411201, China \\ ${ }^{3}$ School of Mechatronics Engineering, Foshan University, Foshan 528000, China \\ ${ }^{4}$ College of Mechanical and Vehicle Engineering, Hunan University, Changsha 410082, China \\ ${ }^{5}$ Hunan Institute of Metrology and Test, Changsha 410014, China
}

Correspondence should be addressed to Kuanfang He; hnkjdxhkfpyf@163.com

Received 5 January 2020; Revised 24 May 2020; Accepted 27 May 2020; Published 18 July 2020

Academic Editor: Petr Hájek

Copyright (C) 2020 Yanfeng Peng et al. This is an open access article distributed under the Creative Commons Attribution License, which permits unrestricted use, distribution, and reproduction in any medium, provided the original work is properly cited.

A novel adaptive signal decomposition algorithm, broadband mode decomposition (BMD), is proposed for analyzing nonstationary broadband signals. Unavoidable error will occur when applying former time-frequency methods to broadband signals, which is caused by Gibbs phenomenon and the calculation of extrema. To overcome that problem, BMD is proposed by searching in the associative dictionary that contains both broadband and narrowband signals. The procedure of the proposed method is as follows: First, the collected datasets are analyzed by BMD and the composite multiscale fuzzy entropies (CMFEs) of the obtained effective components are calculated. Then, locality preserving projection (LPP) is applied for further feature extraction. Analysis results show BMD is more effective when drawing broadband feature from noise and BMD is adaptive for the quality monitoring of DPMIG welding.

\section{Introduction}

Research on signal processing methods for extracting useful feature from noised metal inert gas (MIG) welding signals has been a research hotspot in welding quality estimation [1]. In the MIG welding procedure, the energy put into the welding line is mainly affected by the electrical inputs, which will immediately impact the quality. Thus, the feature embedded in the electrical data can reflect the quality of MIG welding [2]. Nevertheless, the high frequency electric data of double pulse metal inert gas (DPMIG) are modulated by the low frequency pulse frequencies and mixed with severe noise signals. Feature information which can reflect the welding quality usually is narrow-band signals, and the pulse signals such as square signals and sawtooth signals with "sharp corners" are broadband signals as their frequency bands are infinite [3]. Therefore, in order to extract narrowband feature which contains the quality information of welding from pulse signals and noise signals, a suitable time-frequency method should be applied.

Many time-frequency analyses algorithms have been used for extracting feature from noise in engineering fields [3-5]. These adaptive time-frequency analysis algorithms can be classified into two categories: FT based algorithms and non-FT based algorithms [6-8]. The FT based algorithms including the variational mode decomposition (VMD) use the calculations of frequencies to fulfill the data analysis procedure [9]. VMD algorithm is an iteration 
procedure using Wiener filter for denoising. VMD was introduced by Dragomiretskiy and Zosso [10] and showed the effectiveness in analyzing nonstationary industrial signal compared with other algorithms [11-13]. Non-FT based algorithms including empirical mode decomposition (EMD) separate the original data into intrinsic mode functions (IMFs) through the calculation of the extrema [14-16]. Ensemble empirical mode decomposition (EEMD) was introduced by $\mathrm{Wu}$ et al. to increase the denoising ability of EMD by mixing various levels of noise in the data and calculating the average of the generated IMFs [17-19]. Researches have demonstrated the advantage and validity of EEMD and VMD nearly $[11,15]$; the two methods are selected to compare with the BMD algorithm.

The former time-frequency analysis methods are widely applied for extract feature from complex nonstationary signals. However, problems will appear when applying to broadband data as the bandwidth is infinite. Firstly, for FT based algorithms, the substance of the methods is multiple adaptive filters. Nevertheless, the Gibbs phenomenon indicates the high frequencies of broadband signals will attenuate or vanish after smoothing [20], which may generate a range of fluctuations at the mutant points of the decomposed results. Secondly, for non-FT based algorithms, the original data is decomposed into a series of "smooth" narrowband IMFs by computing the extrema envelops utilizing interpolating functions. Thus, an inevitable error will be generated when handling the broadband signals.

For extracting useful information from complicated signals containing typical broadband modes and noise components, the broadband mode decomposition (BMD) algorithm is proposed. In BMD algorithm, an associative dictionary containing common broadband data, such as sawtooth, square, and narrowband data, is constructed. Then, the sparse solution is gained by seeking in the associative dictionary applying an optimization method. The BMD method utilizes a regulated smooth operator as the optimal object. The initial phase, the frequency, and the amplitude of common broadband data are used as the optimal arguments. The artificial chemical reaction optimization algorithm (ACROA) [21], which was introduced by Alatas, is employed for the solution of the optimal problems, and the sparse separation outcomes are gained during the optimal procedure. Compared with previous signal decomposition algorithms, BMD is suitable for extracting broadband features and narrowband features from noisy complex nonstationary signals.

Broadband data including sawtooth and square data are used as the input voltage and current signals of DPMIG welding. As stated above, an inevitable error will be generated while dealing with broadband electric signals of inverter power sources when using previous adaptive signal decomposition signals. Thus, an algorithm for dealing with broadband data such as the introduced BMD algorithm is required. In this paper, the collected welding input electric datasets are applied for evaluating the validity of BMD.

To evaluate the quality of DPMIG welding, suitable parameters should be calculated for the representation of features. Nevertheless, it is worth noting that not all the features are useful for evaluating the quality. Thus, suitable algorithms should be applied for merging the effective information from the mixed broadband and noisy modes, as well as reducing the feature dimension. Many developed feature extraction methods containing linear discriminant analysis (LDA), principal component analysis (PCA), Laplacian eigenmaps (LE), and locally linear embedding (LLE) [22-25] have been introduced to carry out the goal. Locality preserving projections (LPP) [26] was introduced by $\mathrm{He}$ et al. It can produce projective linear mapping which has the essential feature of the datasets in a lowdimensional space. LPP has been demonstrated to be suitable for complex data processing in a plenty of researches [27-29]. Therefore, it is selected for fusing the feature vectors generated from the obtained MIG welding electric datasets.

The remaining contents are organized as below. In Section 2, the existing disadvantages in analyzing the broadband data are given out. The iteration procedure of the BMD method is given in Section 3. In Section 4, the basic theories of LPP are stated. In Section 5, the collected experimental datasets of aluminum alloy DPMIG welding input voltage and current data are tested by the introduced algorithm. The conclusion is given out at last.

\section{Existing Problems in Broadband Signal Analysis}

2.1. VMD Method. The Fourier progression unfolds the data into a series of sinusoidal components. The square signal is taken as an illustration of broadband data; the Fourier progression is as follows:

$$
\text { square }(t)=\sum_{i=1}^{+\infty} \frac{1}{2 i-1} \sin [(2 i-1) t] .
$$

As the bandwidth of a filter is restricted, the high frequencies of broadband signals will attenuate or vanish after filtering, which may induce a range of fluctuations at the mutant points of the decomposed results. When the bandwidth of the used filter is wider, the obtained filtering results are more accurate. This is named as Gibbs phenomenon [20].

VMD decomposes a dataset into a series of band limited IMFs by using multiple Wiener filters. The filter parameters including the bandwidth and center frequency are gained by searching for the variational modes. As the substance of the methods is adaptive filters, it will be influenced by the Gibbs phenomenon when processing with broadband datasets. The iteration procedure for obtaining IMFs is as follows [10]:

(1) Calculate the mathematical formula of $\operatorname{IMF}_{i}(n)$ by using the Hilbert transform:

$$
\left[\delta(n)+\frac{j}{\pi n}\right] * \operatorname{IMF}_{i}(n)
$$

where $\delta$ is the Dirac distribution and denotes convolution. 
(2) Compute the center frequency $\omega_{i}$ of $\operatorname{IMF}_{i}(n)$; transform the formula in equation (2) to the frequency domain corresponding to $\omega_{i}$ as follows:

$$
\left\{\left[\delta(n)+\frac{j}{\pi n}\right] * \operatorname{IMF}_{i}(n)\right\} e^{-j w_{i} n}
$$

(3) An optimization problem is constructed as equation (4); the optimal object is the $L^{2}$ norm of the gradient value of the frequency domain formula. The separated results are gained by solving the optimization problem using the alternate direction multiplier method:

$$
\begin{array}{ll}
\min _{\left\{I M F_{i}(n)\right\},\left\{w_{i}\right\}} & \sum_{i}\left\|\partial_{t}\left\{\left[\delta(n)+\frac{j}{\pi n}\right] * \operatorname{IMF}_{i}(n)\right\} e^{-j w_{i} n}\right\|^{2} \\
\text { S.T. } & x(n)=\sum \operatorname{IMF}_{i}(n) .
\end{array}
$$

2.2. EEMD Method. By the continual extraction of the mean lower and upper envelopes applying the interpolating method, EMD separates the original data into a series of IMFs. The iteration procedure of EMD is as follows [8]:

(1) Set $h_{i}(n)=x(n), i=1$, and $j=1$.

(2) Interpolate the extrema of $h_{i}(n)$ using the interpolating method to gain the lower envelope and upper envelope $m_{u}(n)$ and $m_{l}(n)$; then calculate $h_{i}(n)$ :

$$
h_{i+1}(n)=h_{i}(n)-\frac{m_{u}(n)+m_{i}(n)}{2} .
$$

(3) Step (2) is repeated until $h_{i+1}(n)$ accords with the condition of the IMFs [8]. The $j$ th IMF is obtained:

$$
\operatorname{IMF}_{j}(n)=h_{i+1}(n) .
$$

(4) Set $r(n)=x(n)-\mathrm{IMF}_{j}(n)$; set the residue $r(n)$ to be the updated $x(n)$. Steps (1)-(3) are repeated until $r(n)$ is monotonic data. The separated results are expressed as follows:

$$
x(n)=\sum_{j=1}^{N} \operatorname{IMF}_{j}(n)+r(n) .
$$

EMD is suitable for processing nonstationary datasets [14]. Nevertheless, mode mixture is one of the main disadvantages existing in EMD algorithm, which may induce serious error between the useful information when the data is strongly interfered by noise. Thus, to increase the mode mixing resisting and denoising ability, EEMD is introduced through the addition of various levels of noise in the data and calculating the average of the generated IMFs [15]. The additional noise datasets are irrelevant; thus the averaging procedure of EEMD can offset part of the noise.

Non-FT based algorithms including EMD and EEMD apply the interpolating method to gain the envelope of the extrema. That will induce smooth upper and lower envelopes. Because the obtained IMFs are calculated from the envelopes, the smooth separation results will also be generated when conducting with broadband data.

\section{BMD Algorithm}

3.1. Dictionary. To decompose both the broadband modes and the AM-FM narrowband modes from noisy signals, an associative dictionary containing common broadband data, such as sawtooth, square, and narrowband data, is constructed at first, in which the narrowband dictionary was defined in [30]. The analysis results are gained by seeking in the 3 dictionaries defined as follows:

$$
\begin{aligned}
& \text { Di } c_{1}=\left\{A_{1} \text { square }\left(\omega_{1} n+\theta_{1}, D_{1}\right)\right\}, \\
& \text { Di } c_{2}=\left\{A_{2} \operatorname{sawtooch}\left(\omega_{2} n+\theta_{2}, D_{2}\right)\right\}, \\
& \text { Di } c_{3}=\left\{A_{3}(n) \cos \left(\omega_{3} n+\theta_{3}(n)\right)\right\} .
\end{aligned}
$$

$\theta, \omega$, and $A$ represent the original phase, the angular frequency, and the amplitude, respectively. In $D i c_{3}$, the maximal angular frequency of $A_{3}(n)$ is restricted to be much less than $\omega_{3} . \theta_{3}(n)$ is restricted to be a slowly changing signal to guarantee the elements in $\mathrm{Dic}_{3}$ are narrowband [30].

3.2. BMD Iteration Procedure. The BMD algorithm is introduced to solve the existing disadvantages of extracting broadband features when using previous signal decomposition algorithms, including the Gibbs phenomenon in FT based algorithms and the interpolating of extrema in non-FT based algorithms. In BMD, the associative dictionaries are established at first. Then, the sparse decomposition results are gained by seeking in the dictionaries. BMD utilizes a normalized smoothness objective function as the optimal object. The parameters of the typical broadband datasets are used as the optimal parameters. The ACROA is used for the solution of the optimal problems [21]. In the end, both the broadband $I M F$ s and the narrowband $I M F$ s can be separated from noisy signals by using the optimal procedure. The flow chart of BMD is illustrated in Figure 1. The main iteration steps are designed as follows:

(1) $r_{0}(n)$ is set to equal $x(n)$

(2) Separate the direct-current (DC) signal:

$$
\begin{aligned}
\operatorname{IMF}_{0}(n) & =i f f t\left[\widehat{r}_{0}(1)\right], \\
r_{1}(n) & =x(n)-\operatorname{IMF}_{0}(n) .
\end{aligned}
$$

$\widehat{r}_{0}(k)$ is the FT of $r_{0}(n)$; if $f t\left[\widehat{r}_{0}(1)\right]$ is the IFT of $\widehat{r}_{0}(1)$.

(3) $i$ is set to equal 1 ; the optimal problem P1 is constructed as follows: 


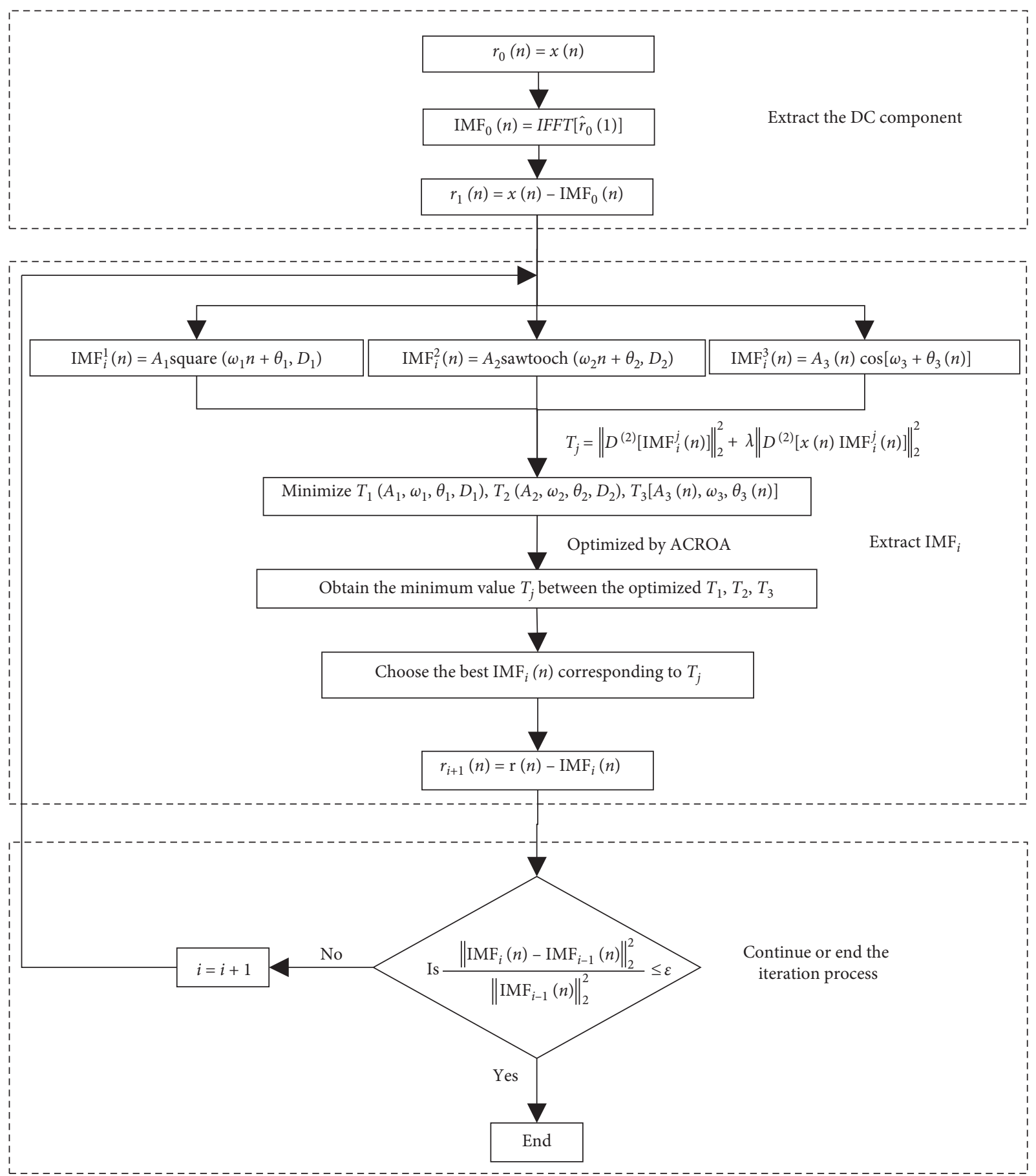

FIgURE 1: The iteration procedure of BMD.

P1: Minimize $T_{1}\left(A_{1}, \omega_{1}, \theta_{1}, D_{1}\right), T_{2}\left(A_{2}, \omega_{2}, \theta_{2}, D_{2}\right), T_{3}\left[A_{3}(n), \omega_{3}(n), \theta_{3}(n)\right]$

S.T.

$$
\begin{aligned}
x(n) & =\sum_{i=0}^{N} \operatorname{IMF}_{i}(n)+\operatorname{res}(n), \quad \operatorname{IMF}_{i}^{j} \in D i c_{j}, \\
T_{j} & =\left\|D^{(2)}\left[\operatorname{IMF}_{i}^{j}(n)\right]\right\|_{2}^{2}+\lambda\left\|D^{(2)}\left[x(n)-\operatorname{IMF}_{i}^{j}(n)\right]\right\|_{2}^{2} .
\end{aligned}
$$

$T_{j}$ is a normalized smooth operator to gain the sparse solution of $r_{0}(n)$ [30], $D^{(2)}$ is the quadratic differential form operator, and $\lambda>0$ is always set to equal 1. 
(4) Solve L1 by using the ACROA to gain the optimal $T_{j(j=1,2,3)}$, find the minimum $T_{j}$, and choose the optimal $\operatorname{IMF}_{i}(n)$ of $T_{j}$. The procedure of the method is introduced in as follows.

(5) Update $r_{i}(n): r_{i+1}(n)=r_{i}(n)-\operatorname{IMF}_{i}(n)$.

(6) If the terminal condition in equation (11) is achieved, terminate the iteration procedure; else, get back to step 3. $\varepsilon$ is a preset parameter which is normally set to be $1 \times 10^{-6}$ :

$$
\frac{\left\|\operatorname{IMF}_{i}(n)-\operatorname{IMF}_{i-1}(n)\right\|_{2}^{2}}{\left\|\operatorname{IMF}_{i-1}(n)\right\|_{2}^{2} \leq \varepsilon} .
$$

3.3. Solution for Optimization Problem P1. In P1, all three dictionaries should be searched by the ACROA. For $D i c_{1}$ and $D i c_{2}$, the ACROA is straightly used, as only the 4 optimal parameters containing $\theta, \omega, A$ and $D$ should be obtained. For $D i c_{3}$, assume the dimension of $x(n)$ is $N \times 1 ; 3 \times N$ optimal parameters must be obtained in every iteration procedure. That will generate a mass of calculation. Thus, the optimal problem of seeking in $\mathrm{Dic}_{3}$ is transformed into obtaining the optimal filter parameters as follows:

(1) Construct a bandpass filter $\chi(k \mid \lambda), \lambda=\left[\omega, \omega_{b}, \omega_{c}\right]$ (shown in Figure 2) is the parameter vector of the bandpass filter, and $\omega, \omega_{b}$, and $\omega_{c}$ determine the decay rate, the bandwidth, and the center frequency of the filter, respectively:

$$
\chi(k \mid \lambda)= \begin{cases}\sin \omega\left[k-\omega_{c}+\omega_{b}+\frac{\pi}{(2 \omega)}\right], & \omega_{c}-\omega_{b}-\frac{\pi}{(2 \omega)} \leq k<\omega_{c}-\omega_{b}, \\ 1, & \omega_{c}-\omega_{b} \leq k \leq \omega_{c}+\omega_{b}, \\ \cos \omega\left(k-\omega_{c}-\omega_{b}\right), & \omega_{c}+\omega_{b}<k \leq \omega_{c}+\omega_{b}+\frac{\pi}{(2 \omega)}, \\ 0, & \text { else. }\end{cases}
$$

(2) Solve the optimization problem P2 by the ACROA:

$$
\begin{aligned}
& \text { P2: Minimize } T_{3}=\| D^{(2)}\left[\text { ifft }\left[\chi(k \mid \lambda) \widehat{r}_{i}(k)\right]\right] \|_{2}^{2} \\
& \quad+\lambda\left\|D^{(2)}\left[r_{i}(n)-i f f t\left[\chi(k \mid \lambda) \widehat{r}_{i}(k)\right]\right]\right\|_{2}^{2} \\
& \text { S.T. } \operatorname{IMF}_{i}^{3}(n)=\text { ifft }\left[\chi(k \mid \lambda) \widehat{r}_{i}(k)\right] .
\end{aligned}
$$

$\widehat{r}_{i}(k)$ is the Fourier transformation of $r_{i}(n)$ and if $f t$ denotes the inverse Fourier transformation.

(3) $\operatorname{IMF}_{i}^{3}(n)$ is calculated by using the optimal parameter $\lambda_{0}$ :

$$
\operatorname{IMF}_{i}^{3}(n)=i f f t\left[\chi\left(k \mid \lambda_{0}\right) \widehat{r}_{i}(k)\right] .
$$

To reduce the calculated quantity of BMD algorithm, the optimization problem P1 in time domain is translated to frequency domain. The representative solution of P1 is

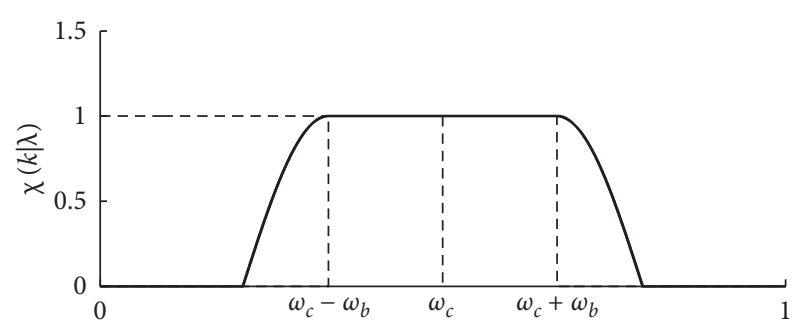

Figure 2: The bandpass filter.

constructed by applying a bandpass filter. The decay rate $\omega$, the bandwidth $\omega_{b}$, and the center frequency $\omega_{c}$ of the filter are optimized during the iteration process. And the optimal objective function $T_{3}$ is used to constrain the obtained results to be effective components. Therefore, the final generated component is obtained by filtering the original signal using the optimized bandpass filter.

3.4. Simulation Analysis. Define square ( $\omega n$, rate) as the square signals, the angular frequency is denoted as $\omega$, and the duty ratio is denoted as rate. The composite signal $x(n)$ of equation (15) contains a square component, an AM-FM sine component, and a noised signal $n(t)$ with the signal noise ratio (SNR) of 3; the sampling rate is $12 \mathrm{kHz}$. The time domain curves of $x(n)$ and its elements are illustrated in Figure 3:

$$
x(n)=\text { square }(20 \pi n, 0.5)+2 \cos (300 \pi n) e^{-2 t^{2}}+n(t) .
$$

For the sake of comparison, $x(n)$ is separated using BMD, EEMD, and VMD, respectively. Figure 4 shows the decomposed results of $x(n)$ by applying VMD. A series of apparent disturbances are shown in the figure, and the broadband feature of the square data is lacking, which is because the intrinsic theory of VMD is adaptive filtering; it will be influenced by Gibbs phenomenon. Figure 5 illustrates the separated results by applying EEMD. It can be seen that the obtained $\mathrm{IMF}_{1}$ corresponding to the square signal seems like a sine signal. Apparently, the obtained useful information is not correct. This is because EEMD uses an interpolating algorithm to gain the extrema envelopes, which will induce smooth IMFs. As the envelopes are calculated to obtain the IMFs, the eventual separated results of EEMD will turn into smooth IMFs when analyzing broadband datasets. Figure 6 illustrates the separated IMFs by using BMD. Therefore, the generated $\mathrm{IMF}_{1}$ is close to the real square component and the mutant feature is maintained.

For the purpose of comparison, the accuracy parameters of the separated IMFs obtained by the 3 algorithms are illustrated in Table 1, and the accuracy parameters containing the error of energy $E_{i}$, the coefficient of correlation $r_{i}$, and the time $T$ are applied [30]. Table 1 illustrates the IMFs obtained by BMD are closer to the square component in $x(n)$. To compare the computation time, the separating procedure is conducted on identical computer. The analysis results indicate the BMD algorithm requires more 


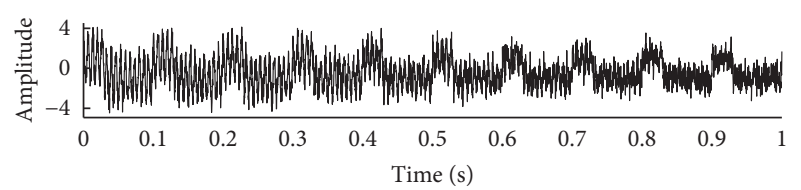

(a)

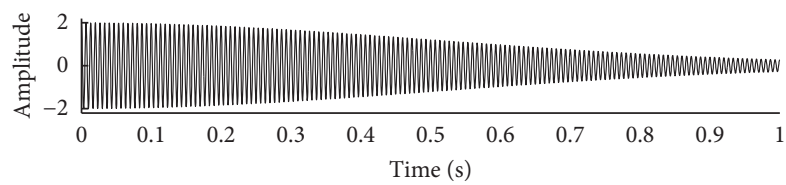

(c)

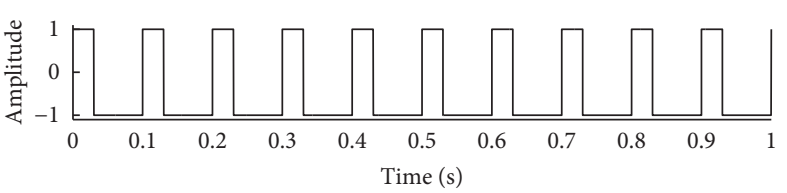

(b)

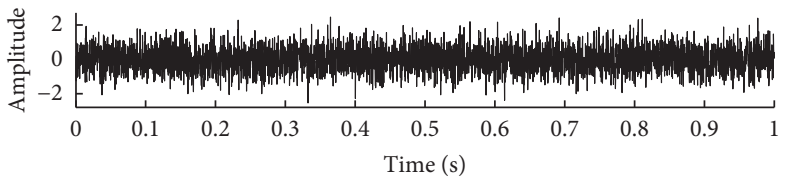

(d)

Figure 3: The time-domain waveforms of $x(n)$. (a) Mixed signal $x(n)$. (b) Square component. (c) AM-FM sinusoidal component. (d) Noise component.

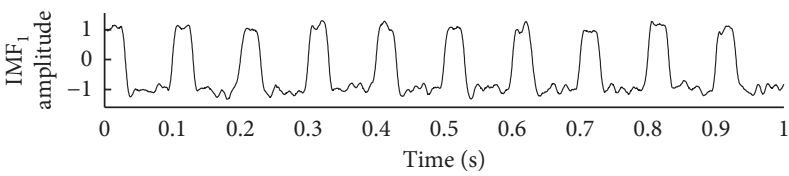

(a)

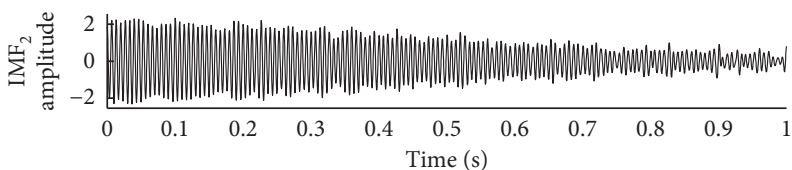

(b)

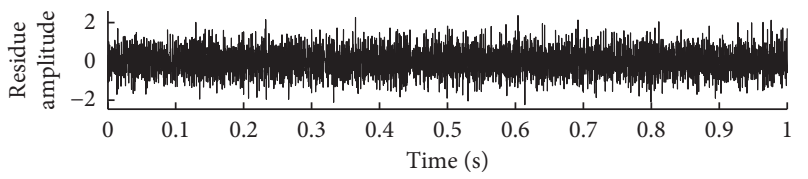

(c)

Figure 4: The decomposition results of $x(n)$ generated by VMD. (a) The IMF corresponding to the square component. (b) The IMF corresponding to the AM-FM sinusoidal component.

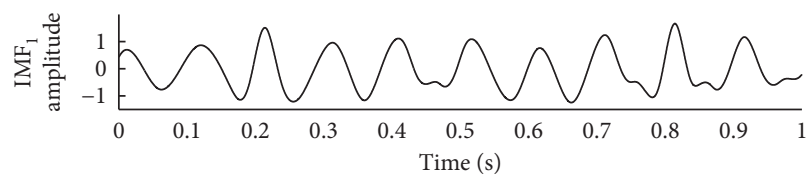

(a)

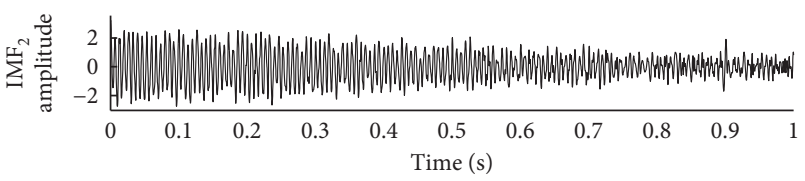

(b)

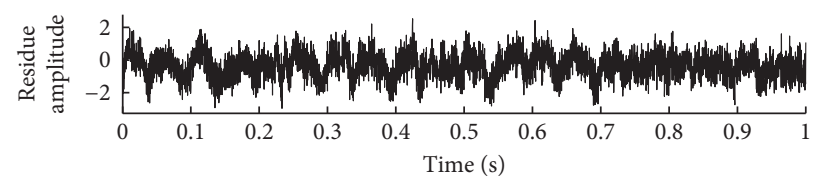

(c)

FIgURE 5: The decomposition results of $x(n)$ generated by EEMD. (a) The IMF corresponding to the square component. (b) The IMF corresponding to the AM-FM sinusoidal component.

computational time compared with EEMD and VMD because of the complicated optimal procedure.

To analyze the performance of BMD in mode mixture phenomenon, a simulation signal $y(n)$ shown in equation (16) is decomposed by VMD, EEMD, and BMD, respectively. To test the robust performance of $\mathrm{BMD}$ in different noise intensity, the SNRs of $y(n)$ are set to be $-10,-5$, and 0 , respectively. The sampling rate is $12 \mathrm{kHz}$. The time domain curves of $y(n)$ and its elements are illustrated in Figure 7:

$$
x(n)=\text { square }(40 \pi n, 0.5)+n(t) .
$$

Figures 8 and 9 show the decomposed results of $y(n)$ by applying VMD and EEMD. In Figure 8, it can be seen that, due to the low SNR, VMD treats the square signal as narrow 


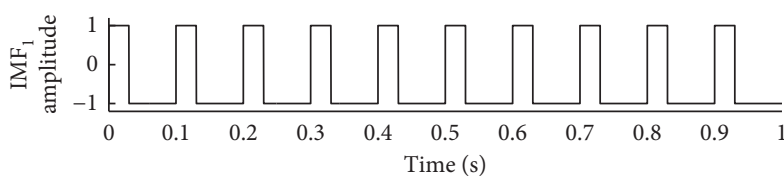

(a)

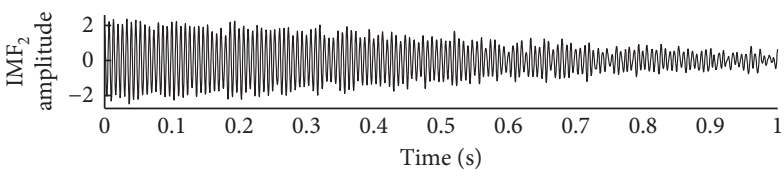

(b)

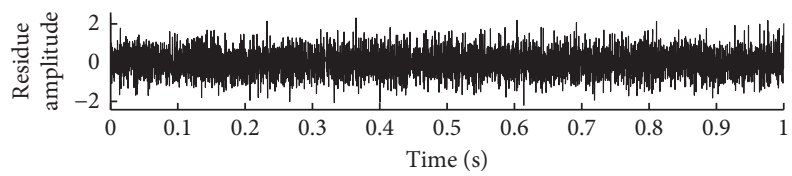

(c)

Figure 6: The decomposition results of $x(n)$ generated by BMD. (a) The IMF corresponding to the square component. (b) The IMF corresponding to the AM-FM sinusoidal component.

TABLE 1: Evaluation parameters of $x(n)$ of the generated components.

\begin{tabular}{lccccc}
\hline Method & $r_{1}$ & $E_{1}$ & $r_{2}$ & $E_{2}$ & $T(s)$ \\
\hline VMD & 0.9615 & 0.0686 & 0.9765 & 0.0464 & 4.7231 \\
EEMD & 0.8014 & 0.7983 & 0.9064 & 0.1783 & 8.9837 \\
BMD & 0.9988 & 0.0020 & 0.9782 & 0.0138 & 36.2351 \\
\hline
\end{tabular}

band signal. Moreover, apparent disturbances are shown in Figure 8(a) when the SNR equals -10 , which is caused by mode mixture. In Figure 9 of the EEMD generated decomposition results, the effective components are still close to sine signals due to the calculation of envelops. Meanwhile, when SNR equals -10 and -5 , there are obvious disturbances mixed by the strong noise. Figure 10 shows the generated decomposition results of BMD. In Figures $10(\mathrm{a})$ and $10(\mathrm{~b}), \mathrm{BMD}$ treats the square component as narrowband signal, which is caused by the low SNRs. Otherwise, the generated components are mixed by other modes, where it can be inferred that when a decomposed component of BMD belongs to the narrowband signal dictionary $\mathrm{Dic}_{3}$, there may still exist mode mixing problem when the noise is strong enough. However, the decomposed results of BMD more approach the real square component when the noise intensity varies, which indicates BMD is more robust to noise. For further analysis, the parameters of the separated IMFs obtained by the 3 algorithms are illustrated in Table 2; it can be easily concluded that although BMD costs more calculation time than the other 2 methods, it is still more robust to noise and behaves better in mode mixing.

\section{CMFE and LPP}

4.1. CMFE. The DPMIG welding quality is mainly determined by the stability of the injected energy, which can be reflected by the nonlinearity degree of the input electrical signals. One of the most effective ways to measure the nonlinearity degree of nonstationary signals is entropy. When the entropy value is bigger, the nonlinearity degree of the collected DPMIG welding electrical signals is higher, which means the welding quality is worse. In the real physical world, the boundaries between different classes may be ambiguous, and it is difficult to determine whether an input pattern totally belongs to a given class or the other. Therefore, fuzzy entropy (FE) was proposed by applying the exponential function and removing the mean of matching template. The fuzzy entropy estimation procedure can be described as follows:

(1) A normalized time series $\{x(i): 1 \leq i \leq N\}$ with length $N$ is considered for the sake of convenience. For given $m, n$, and $r$, a vector set sequence $\left\{X_{i}^{m}, i=1,2, \ldots, N-m+1\right\}$ is constructed in the form as

$$
\begin{aligned}
X_{i}^{m} & =\{x(i), x(i+1), \ldots, x(i+m-1)\}-x_{0}(i), \\
i & =1,2, \ldots, N-m+1,
\end{aligned}
$$

where $X_{i}^{m}$ represents $m$ consecutive $u$ values, commencing with the $i$ th point, and is obtained by removing their baseline:

$$
x_{0}(i)=m^{-1} \sum_{k=0}^{m-1} x(i+k)
$$

(2) For a certain vector $X_{i}^{m}$, the distance $d_{i j}^{m}$ between $X_{i}^{m}$ and $X_{j}^{m}$ is defined as the maximum absolute difference of the corresponding scalar components, namely,

$$
\begin{aligned}
d_{i j}^{m}=d\left[X_{i}^{m}, X_{j}^{m}\right]= & \max _{k \in(0, m-1)}\left\{\mid\left[u(i+k)-u_{0}(i)\right]\right. \\
& \left.-\left[u(j+k)-u_{0}(j)\right] \mid\right\} .
\end{aligned}
$$

(3) Calculate the similarity degree $D_{i j}^{m}$ of $X_{i}^{m}$ and $X_{j}^{m}$ through the fuzzy function $\mu\left(d_{i j}^{m}, n, r\right)$ :

$$
D_{i j}^{m}=\mu\left(d_{i j}^{m}, n, r\right)=e^{-\ln 2\left(d_{i j}^{m} / r\right)^{n}} .
$$

(4) Define function $\varphi^{m}(n, r)$ as 


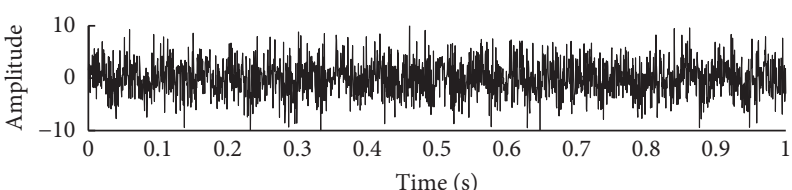

(a)

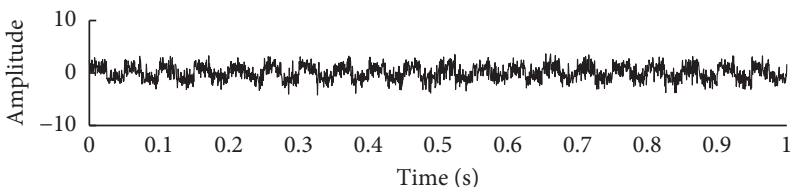

(c)

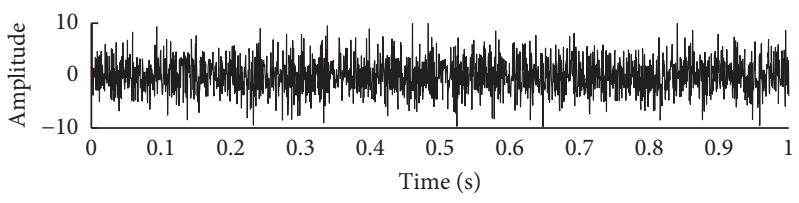

(e)

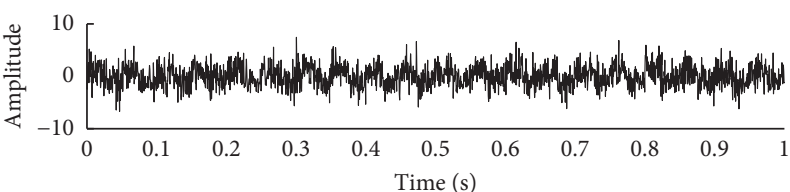

(b)

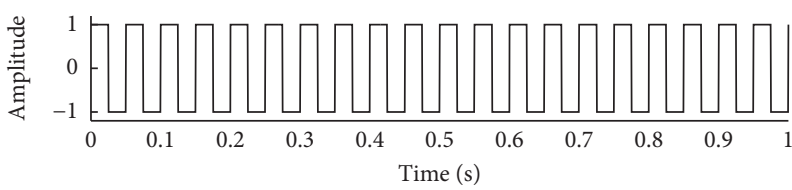

(d)

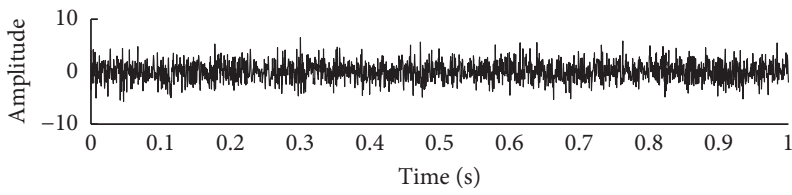

(f)

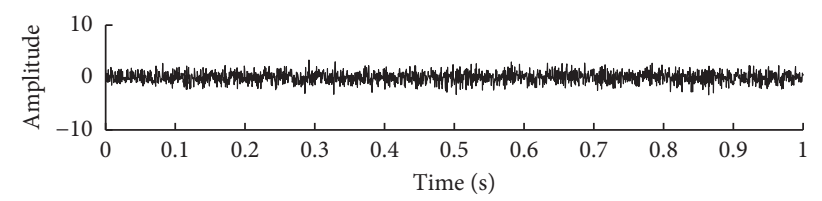

(g)

FIGURE 7: The time-domain waveforms of simulation signal $y(n)$ and its components. (a-c) The mixed signal $y(n)$ as $\mathrm{SNR}=-10,-5$, and 0 , respectively. (d) The square component of $y(n)$. (e-g) The noise components of the signals shown in (a), (b), and (c), respectively.

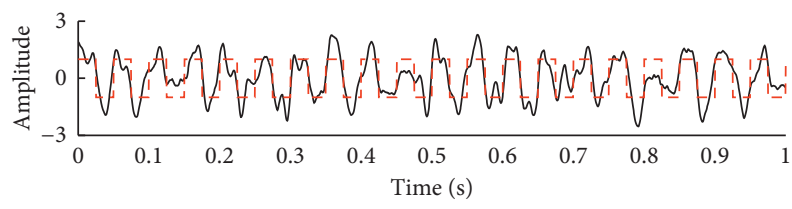

(a)

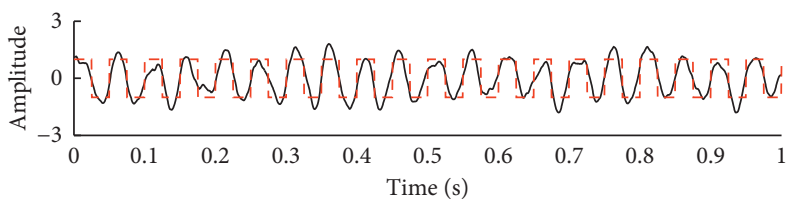

(b)

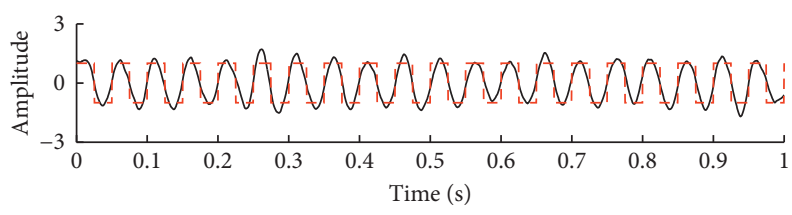

(c)

Figure 8: The decomposed results generated by VMD. (a-c) The generated effective component of $y(n)$ as SNR $=-10,-5$, and 0 , respectively. The red dotted line is the real component and the black solid line is the generated effective component.

$$
\varphi^{m}(n, r)=\frac{1}{N-m} \sum_{i=1}^{N-m}\left(\frac{1}{N-m-1} \sum_{\substack{j=1 \\ j \neq i}}^{N-m} D_{i j}^{m}\right) .
$$

(5) Similarly, for $m+1$, repeat steps (1)-(4) and the function $\varphi^{m+1}(n, r)$ can be obtained as

$$
\varphi^{m+1}(n, r)=\frac{1}{N-m} \sum_{i=1}^{N-m}\left(\frac{1}{N-m-1} \sum_{\substack{j=1 \\ j \neq i}}^{N-m} D_{i j}^{m+1}\right) .
$$

(6) Finally, the FE of the original sequence is defined as the negative natural logarithm of the deviation of $\varphi^{m}(n, r)$ from $\varphi^{m+1}(n, r)$ : 


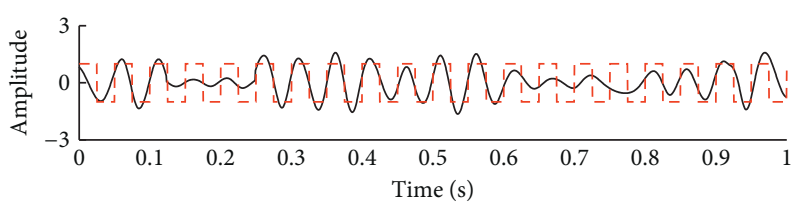

(a)

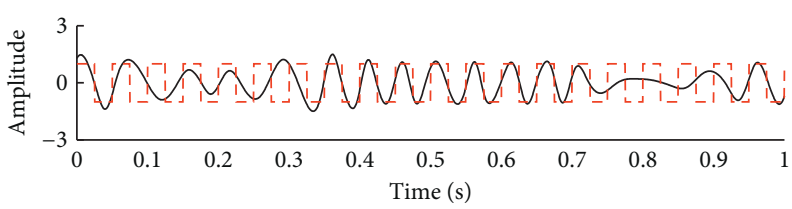

(b)

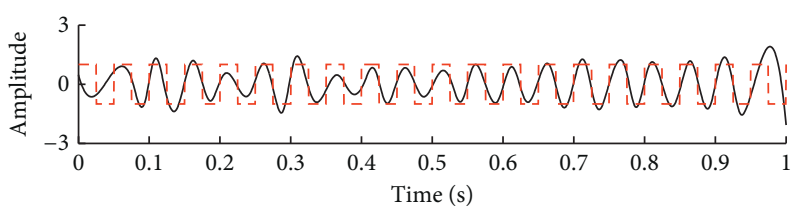

(c)

Figure 9: The decomposed results generated by EEMD. (a-c) The generated effective component of $y(n)$ as SNR $=-10,-5$, and 0 , respectively. The red dotted line is the real component and the black solid line is the generated effective component.

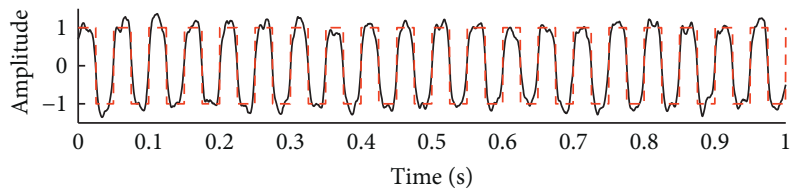

(a)

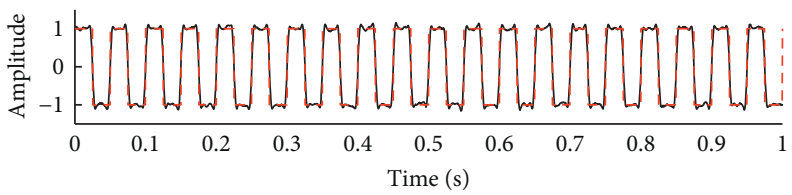

(b)

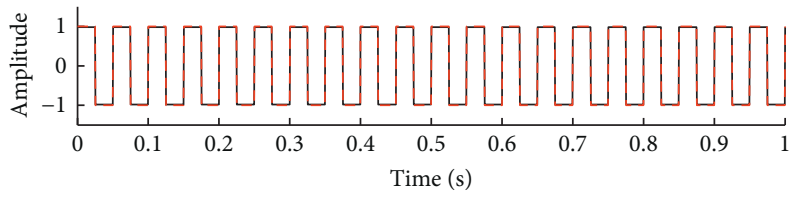

(c)

FIgURE 10: The decomposed results generated by BMD. (a-c) The generated effective component of $y(n)$ as $\mathrm{SNR}=-10,-5$, and 0 , respectively. The red dotted line is the real component and the black solid line is the generated effective component.

$$
\operatorname{FuzzyEn}(m, n, r)=\lim _{N \longrightarrow \infty}\left[\ln \varphi^{m}(n, r)-\ln \varphi^{m+1}(n, r)\right]
$$

When the length of datasets $N$ is finite, $\operatorname{FuzzyEn}(m, n, r)$ can be estimated by the statistic as

$$
\operatorname{FuzzyEn}(m, n, r, N)=\ln \varphi^{m}(n, r)-\ln \varphi^{m+1}(n, r) .
$$

However, FE still lacked a clear statistical and physical significance for short-term time series or coarse-grained series. To alleviate the shortening of the time series in the coarse graining of MFE, CMFE applies the mean multiscale fuzzy entropy (MFE) of different coarse graining time series with the same scale factor as the extracted feature. If the CMFE of a given signal is larger, the mean MFE with a specific scale of different coarse graining time series of the signal is larger, which means that the given signal contains more mode information and is more complicated. To verify the stability of welding quantitatively and intuitively, the CMFE of the collected inverter power source electrical signals is calculated. A larger CMFE means a larger uncertainty in the inverter power source output energy, which will lead to a worse welding quantitatively. The calculation process of CMFE is as follows:
TABle 2: Evaluation parameters of $y(n)$ of the generated components.

\begin{tabular}{lcccc}
\hline Method & SNR & $r_{1}$ & $E_{1}$ & $T(s)$ \\
\hline \multirow{3}{*}{ VMD } & -10 & 0.7733 & 0.4238 & 4.1871 \\
& -5 & 0.8432 & 0.3576 & 3.2324 \\
& 0 & 0.8897 & 0.2626 & 5.7304 \\
\hline \multirow{3}{*}{ EEMD } & -10 & 0.6174 & 1.4908 & 7.4127 \\
& -5 & 0.7033 & 0.8417 & 6.9264 \\
& 0 & 0.8203 & 0.5729 & 7.9327 \\
\hline \multirow{3}{*}{ BMD } & -10 & 0.9041 & 0.1292 & 40.5328 \\
& -5 & 0.9377 & 0.0604 & 35.9863 \\
& 0 & 0.9721 & 0.0114 & 38.4571 \\
\hline
\end{tabular}

(1) Suppose a signal function $x(n)$, set the number of data points as $N$, and calculate $y_{k}^{(\tau)}=$ $\left\{y_{k, 1}^{(\tau)}, y_{k, 2}^{(\tau)}, \ldots, y_{k, j}^{(\tau)}\right\}$ of different coarse-grained time series with scale factor as $\tau$. $y_{k}^{(\tau)}$ is the kth coarsegrained time series of $x(n)$ with the scale factor of $\tau$ :

$$
y_{k, j}^{(\tau)}=\frac{1}{\tau} \sum_{i=(j-1) \tau+k}^{j \tau+k-1} x_{i}, \quad 1 \leq j \leq \frac{N}{\tau}, 1 \leq k \leq \tau .
$$


(2) Secondly, the results of fuzzy entropy of each coarsegrained time series $y_{k}^{(\tau)}$ obtained by calculation were averaged to obtain the coarse-grained time series CMFE of the scale factor $\tau$.

$$
\operatorname{CMFE}(x(n), \tau)=\frac{1}{\tau} \sum_{k=1}^{\tau} \operatorname{FuzzyEn}\left(y_{k}^{(\tau)}\right) .
$$

4.2. LPP Algorithm. After the evaluating parameters are calculated from the electric datasets, the feature with the dimension of $M$ is obtained. Nevertheless, the obtained features usually contain redundant information and are high dimensional. Therefore, suitable algorithm should be used for the useful information extraction and the feature dimension reduction. PCA is a widely applied algorithm for the reduction of dimension $[28,29]$, which means increasing the global feature and lack of the ability for maintaining the local structure. Thus, LPP algorithm which can extract the local feature of the given datasets is used for further feature extraction in this article.

LPP maintains the local feature through the extraction of the approximation of the given dataset. $F=\left\{f_{1}\right.$, $\left.f_{2}, \ldots, f_{N_{i}}\right\}$ is set to be the feature vectors. $N_{i}$ and $J$ are the amount of the feature vectors and the data samples. LPP seeks a projection matrix to reduce the dimensions of the feature vectors into $F=\left\{f_{1}^{\prime}, f_{2}^{\prime}, \ldots, f_{N_{i}}^{\prime}\right\} ; J^{\prime}\left(J^{\prime} \ll J\right)$ is the dimension of the fitted feature vector $F^{\prime}$. For choosing a map like that, the object is constructed as follows [28, 29]:

$$
\min \sum_{i, j=1}^{N_{i}}\left\|f_{i}-f_{j}\right\|^{2} S_{i, j}
$$

The parameter $\mathbf{S}$ is obtained by the nearest neighborhood and induces a punishment when the neighbor area $f_{i}$ is far away from $f_{j}$. The formula of $\mathbf{S}$ is as follows:

$$
S_{i, j}=\left\{\begin{array}{l}
\exp \left(\frac{-\left\|f_{i}-f_{j}\right\|^{2}}{\left(2 \sigma^{2}\right)}\right), \quad f_{i} \text { or } f_{j} \text { is between the } k \text { nearest neighbors of } f_{j} \text { or } f_{i}, \text { respectively, } \\
0
\end{array}\right.
$$

$k$ represents the "locality" degree of the local area and $\sigma$ shows the major kernel value. The objective functions are optimized to make sure $f_{j}$ is near $f_{i}$; thus, $f_{j}^{\prime}$ is also near $f_{i}^{\prime}$. Therefore, the local feature is maintained. Set $f_{i}^{\prime}=a^{T} f_{i}$; then, the optimal solution is altered as follows:

$$
\begin{aligned}
J_{l}(a) & =\frac{1}{2} \sum_{i, j=1}^{N_{i}}\left\|f_{i}^{\prime}-f_{j}^{\prime}\right\|^{2} S_{i, j}=\frac{1}{2} \sum_{i, j=1}^{N_{i}}\left\|a^{T} f_{i}-a^{T} f_{j}\right\|^{2} S_{i, j} \\
& =a^{T} F(D-S) F^{T} a=a^{T} F L F^{T} a .
\end{aligned}
$$

A Laplacian matrix $L=D-S$ is constructed. The bigger $D_{i i}$ obtains a more important $f_{i}^{\prime}$. Furthermore, the restriction constraint is set up as follows:

$$
F^{\prime} T D F^{\prime}=1 \Longrightarrow a F D F^{T} a=1 \text {. }
$$

The optimal problem is constructed as follows:

$$
\begin{array}{ll}
\min _{a} & a F L F^{T} a \\
\text { subject to } & a F D F^{T} a=1 .
\end{array}
$$

The constrained optimal object function can be transformed into an eigenvalue question by using Lagrange multiplier algorithm. The transform matrix is gained as follows:

$$
F L F^{T} a=\lambda F D F^{T} a
$$

where $\lambda$ represents the weight value. The dimension reduction formula is as follows:

$$
F^{\prime}=A^{T} F \mathbf{A}=\left[a_{1}, a_{2}, \ldots, a_{l}\right]^{T},
$$

where $\mathbf{A}$ is a matrix with the dimension of $J \times J^{\prime}$. The input vector $F$ is transformed before a dimension reduced vector $F^{\prime}$ is gained. The local useful information is maintained by $F^{\prime}$ and the feature is extracted. The optimal linear local structure is searched in the matrix. Therefore, LPP is adaptive for noisy signals and isolated feature values as the local structure is maintained.

PCA is applied to extract the subspace of maximal difference. Therefore, it can maintain the global information. However, the useful features of the local structure of experimental signals like electric datasets are more meaningful. In addition, LPP has been identified to be adaptive for dealing with the electric datasets. At the same time, researchers have made comparisons between PCA and LPP by using various industrial datasets; the analysis results have demonstrated that PCA performed better in the useful information extraction of features. Therefore, LPP algorithm, which can maintain the useful information, is used in the introduced BMD algorithm for extracting effective feature better. In this paper, the CMFEs of the first two decomposed components are applied as the calculated 
parameter of the analyzed signals; the number of the generated parameters is determined by the number of the scale factor $\tau$. In the experimental analysis part, $\tau$ is set to 1 , 2,3 , and 4 as we find the parameters become more chaos when $\tau$ is bigger than 4 . Therefore, there are 8 generated CMFE parameters with different $\tau$. Then, the CMFE parameters are fused into one parameter vector by LPP to merge the effective information.

\section{Experimental Analysis}

5.1. Data Collection. The experimental electric datasets are collected from the built aluminum alloy DPMIG welding experimental platform illustrated in Figure 11. The experimental platform applies a DPMIG welding machine and a weld robot to proceed with the welding procedure. The aluminum welding machine model is DPMIG-5, which is responsible for setting welding process parameters such as welding peak voltage, welding base value voltage, welding peak current, welding base value current, duty cycle, welding frequency, and the starting and stopping welding experiments. The robot includes the welding robot ABB IRB1410 and the robot controller to control welding speed and walking direction. The data acquisition system consists of a sensor box, an acquisition box, a computer, and a communication cable, which is used to store welding arc data with high speed and accuracy. The wire feeding mechanism includes a wire feeding disc and a wire feeding controller, which can provide two kinds of wire feeding modes for selection: manual wire feeding and automatic wire feeding. In the welding process experiment of argon gas storage tank, inert gas is provided during the transportation of welding wire to isolate the air outside the welding area and prevent oxidation.

The scantling of the aluminum alloy is $0.25 \mathrm{~m} \times 0.2 \mathrm{~m} \times 0.005 \mathrm{~m}$. The argon flow rate is $0.2 \mathrm{~L} / \mathrm{s}$, and the semidiameter of the welding stick is $0.6 \mathrm{~mm}$. The datasets are obtained by using electrical transducers. To test the validity of BMD in various operating conditions, 4 experiments with various current frequency, duty cycle, and welding speed are applied. A 48-second data collection procedure is conducted in each experiment, and the sample frequency is set to be $12 \mathrm{kHz}$. Table 3 shows the experimental parameters. Figure 12 shows the captured electrical datasets of the 4 experiments. The welding appearances are shown in Figure 13. The welding appearance of dataset 3 illustrated in Figure 13(c) has apparent incomplete-penetration and fusion caused by the arc breaking of the input broadband current data.

5.2. Experimental Signal Decomposition. One-second current data in dataset 3 is chosen for the comparison of VMD, EEMD, and BMD, and the time domain waveform of the signal is illustrated in Figure 14. The original signal is decomposed into 4 IMFs and a residual component. The feature corresponding to the quality of welding is extracted from the input broadband and noisy disturbance. Thus, the useful components, the input broadband square component, and the noisy components should be separated. The generated results of VMD are illustrated in Figure 15. The results indicate that VMD is unable to differentiate the useful components and broadband square components, which implies it lacks the ability of separating broadband and narrowband components. The generated results of VMD are illustrated in Figure 16. The IMFs generated by EEMD are quite distinct from the input square signal as it handles the broadband square signals to be sinusoidal signals that will generate serious mode confusion. The generated results of BMD are illustrated in Figure 17. The $\mathrm{IMF}_{1}$ of BMD is similar to the input square signal with the parameters of Table 3, and the $\mathrm{IMF}_{2}$ maintains the mutant feature of the quality of DPMIG welding.

5.3. LPP Feature Extraction. Each of the four datasets is divided into 48 equal samples; each sample contains onesecond signal. All the samples are decomposed by VMD, $\mathrm{BMD}$, and EEMD, respectively. Then, the CMFEs of the generated effective components are calculated to verify the stability of welding quantitatively and intuitively (the scale factor $\tau$ is set to $1,2,3,4)$. Then, the features are fused by LPP, the fused feature vectors are shown in Figures 18-20, and the mean CMFEs in each experiment are shown in Table 4. It can be seen in Figure 20 and Table 4 that the CMFEs generated by applying BMD become larger when the welding speed increases, which means that the welding quality is worse. This is because the electrical energy becomes more dispersed with the increase in the welding speed. In addition, the larger pulse frequency matches better with the welding speed, and the arc energy becomes more stable. Thus, the CMFEs of dataset 3 are larger than that of dataset 4. However, the CMFEs obtained by BMD and EEMD are confusing, which further indicates the extracted feature is disturbed by other modes from the broadband signal and noise. To compare the computation time, the mean time cost parameters of the analysis procedure of the 4 datasets by applying the three adaptive decomposition methods combined with CMFE and LPP are given out in Table 4 . The analysis results show that it still costs more time when applying BMD method to the experimental signals.

The simulated and experimental analyzing results indicate BMD exceeds the other two methods in both the precision and denoising performance. Furthermore, in the experimental analysis, BMD performed better when combined with LPP in the quality feature extraction of aluminum alloy DPMIG welding. 


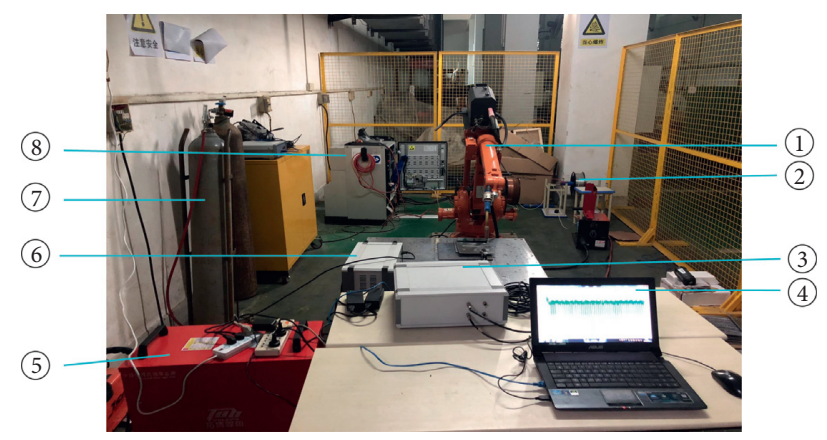

FIGURE 11: The aluminum alloy DPMIG welding test bed. (1)-(8) are the welding robot, the wire feeder, the acquisition box, the computer, the welding machine, the electrical signal sensor box, the argon gas storage tank, and the inverter power source.

Table 3: Parameters of the welding experiment.

\begin{tabular}{lcccc}
\hline Dataset & Welding speed $(\mathrm{mm} / \mathrm{s})$ & Inputting current & \\
Duty cycle (\%) & Peak-to-peak value $(\mathrm{A})$ & 50 & 2 \\
\hline 1 & 1 & 50 & 50 & 2 \\
2 & 1.5 & 50 & 50 & 2 \\
3 & 5 & 50 & 50 & 5 \\
4
\end{tabular}

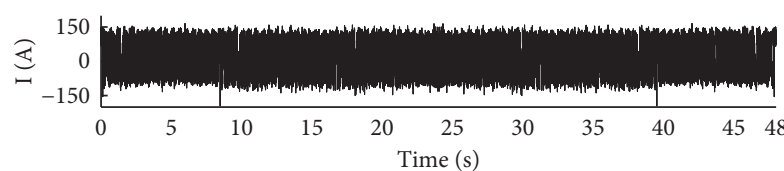

(a)

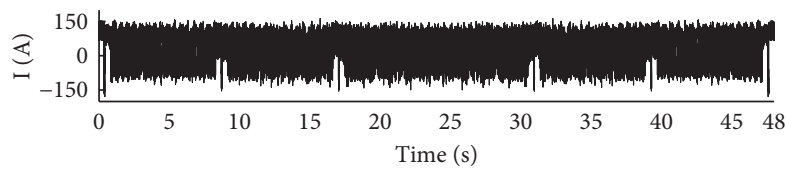

(c)

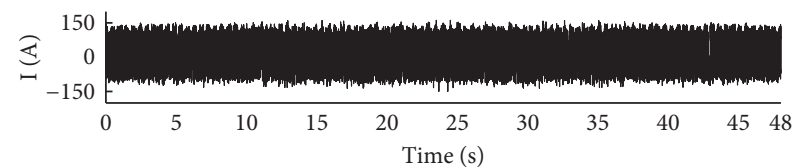

(b)

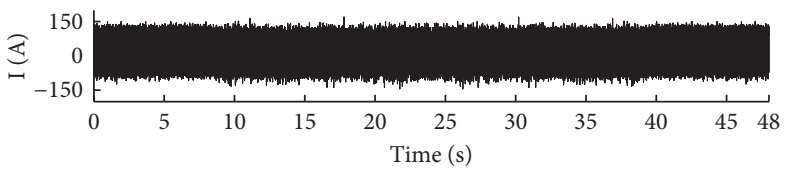

(d)

FIGURE 12: The collected inverter power source output current signals. (a-d) The time-domain waveform of datasets $1-4$, respectively.

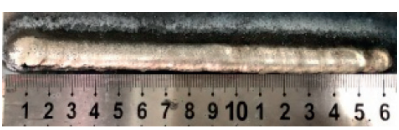

(a)

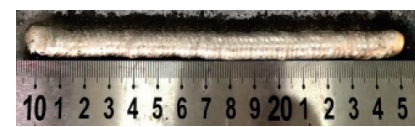

(b)

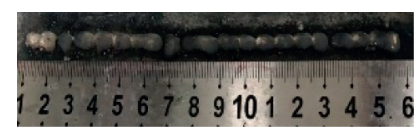

(c)

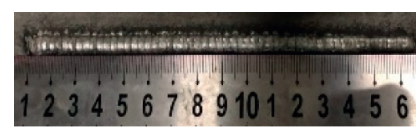

(d)

Figure 13: The appearance of the weld shapes. (a-d) Photos of the weld shapes corresponding to datasets $1-4$, respectively.

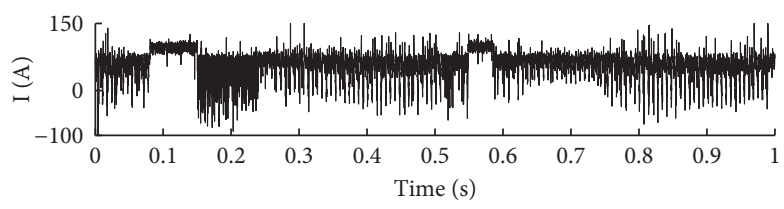

FIgURE 14: The selected experimental signal of dataset 3. 


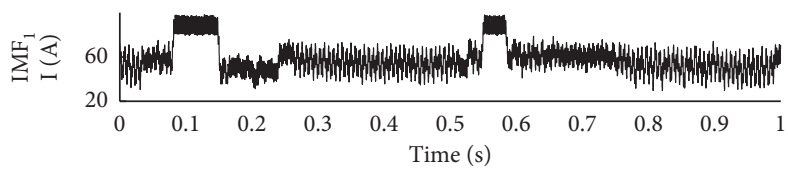

(a)

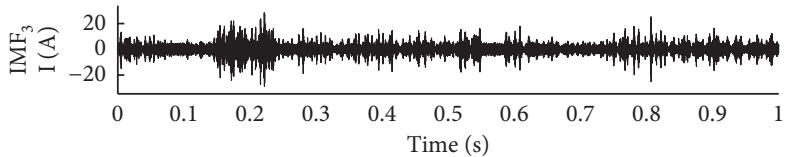

(c)

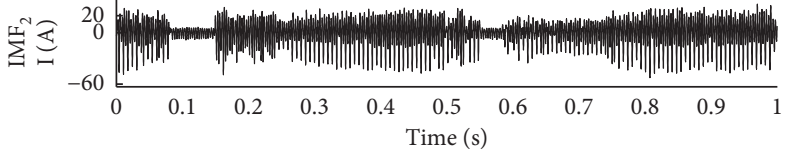

(b)

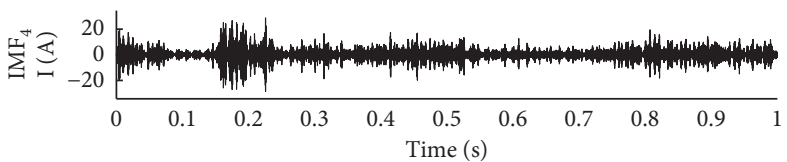

(d)

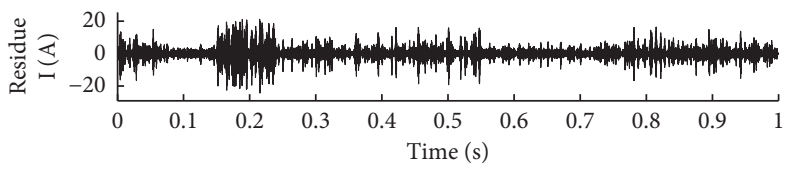

(e)

Figure 15: Decomposition results generated by VMD.

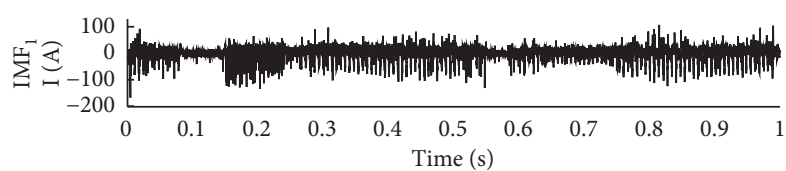

(a)

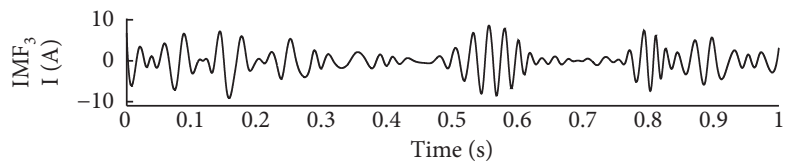

(c)

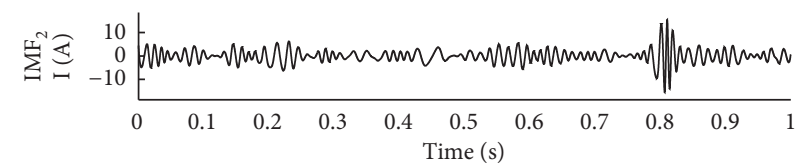

(b)

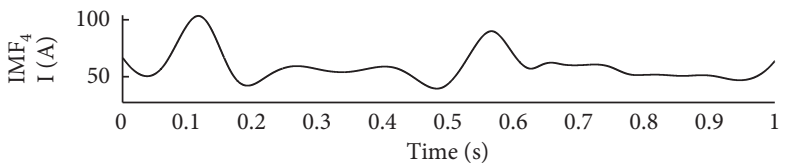

(d)

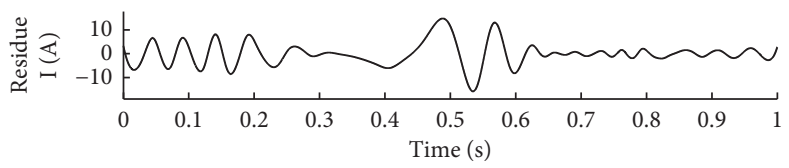

(e)

FIgURE 16: Decomposition results generated by EEMD.

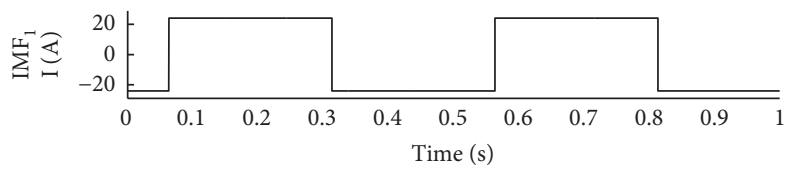

(a)

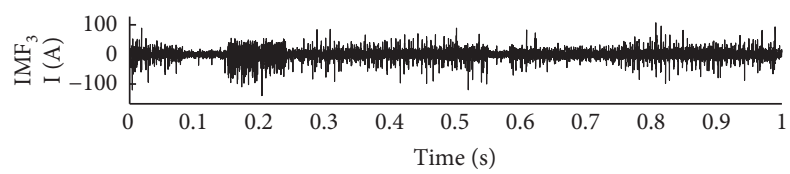

(c)

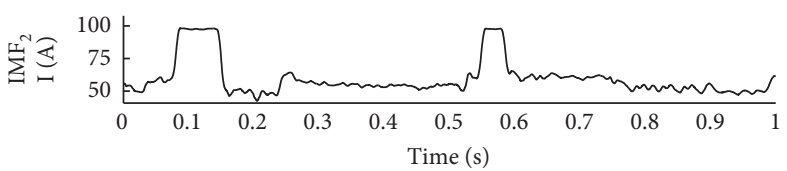

(b)

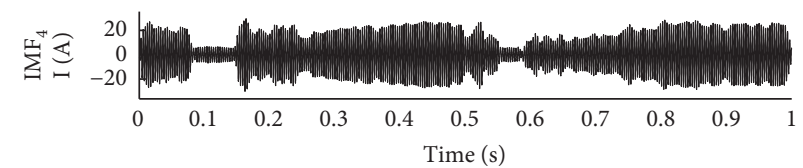

(d)

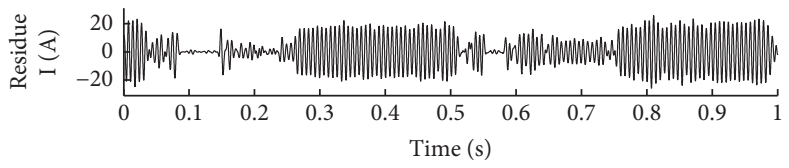

(e)

Figure 17: Decomposition results generated by BMD. 


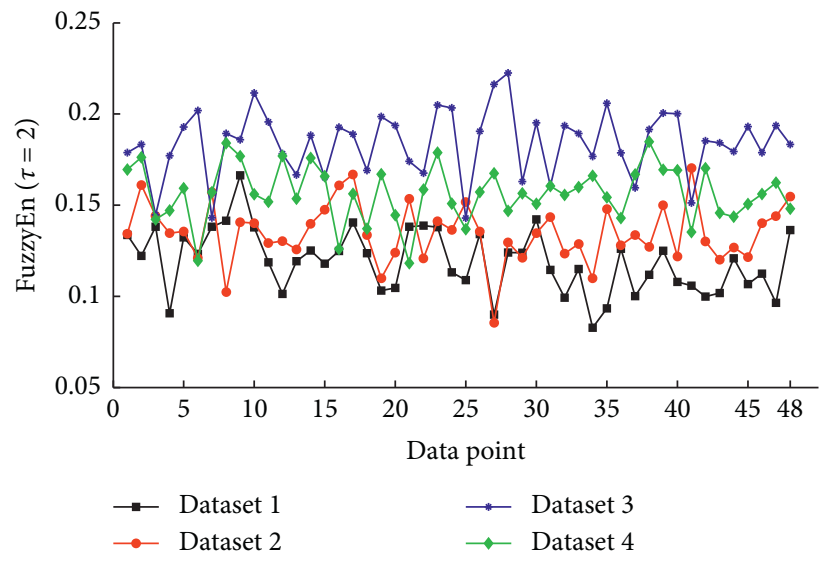

FIgURE 18: The LPP-fused CMFEs of the VMD-generated results.

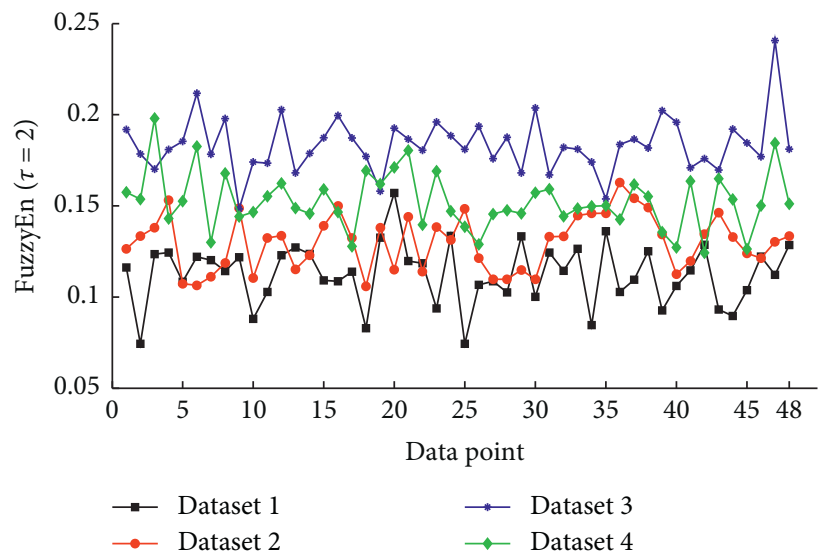

FIGURE 19: The LPP-fused CMFEs of the EEMD-generated results.

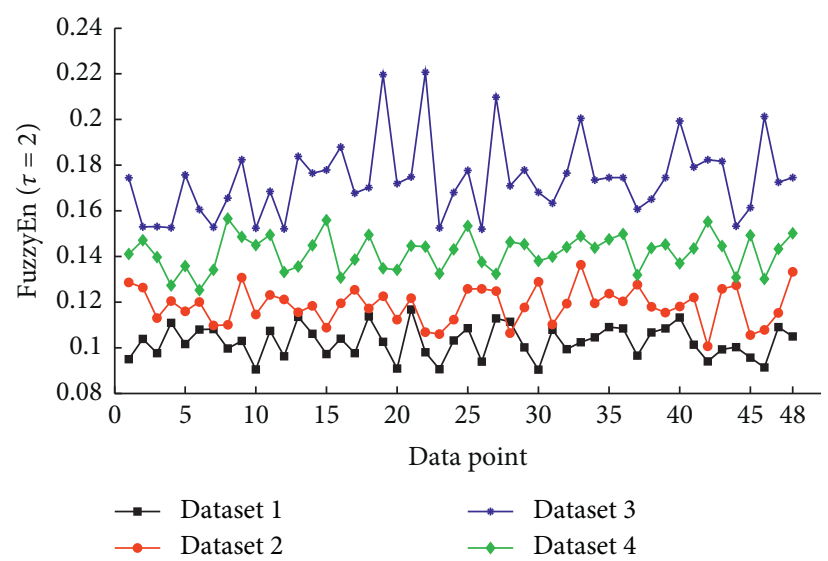

Figure 20: The LPP-fused CMFEs of the BMD-generated results.

TABLE 4: The mean fused CMFEs and the mean time cost of the generated results.

\begin{tabular}{lccccc}
\hline \multirow{2}{*}{ Method } & \multicolumn{2}{c}{ CMFE } & & $T(s)$ \\
Dataset 1 & Dataset 2 & Dataset 3 & Dataset 4 & The mean time cost of the 4 datasets \\
\hline VMD & 0.1107 & 0.1395 & 0.1821 & 0.1536 & 213.1275 \\
EEMD & 0.1145 & 0.1341 & 0.1937 & 0.1547 & 410.2391 \\
BMD & 0.0997 & 0.1191 & 0.1654 & 0.1322 & 1315.5237 \\
\hline
\end{tabular}




\section{Conclusions}

Combined with LPP, the BMD algorithm is introduced for the quality feature extraction of nonstationary broadband electrical signals of aluminum alloy double pulse metal inert gas (DPMIG) welding, and monitoring the influence of the electrical signals on the arc stability and welding appearance. The disadvantages of previous time-frequency algorithms in FT based and non-FT based algorithms induced by the calculation of extrema and Gibbs phenomenon can be alleviated by seeking in the dictionary using ACROA. The superiority of BMD is tested by simulated and experimental data. Analysis results indicate that the proposed method can precisely draw the broadband useful features and evaluate the quality of aluminum alloy DPMIG welding. The proposed method is tested by aluminum alloy DPMIG welding electrical signals. BMD algorithm can also be applied to analyze other broadband industrial data. Nevertheless, the calculation of BMD is still relatively large because of the optimization procedure. Thus, fast iteration algorithms of BMD should be studied in the future.

\section{Data Availability}

All data included in this study are available upon request to the corresponding author.

\section{Conflicts of Interest}

The authors declare that they have no conflicts of interest.

\section{Acknowledgments}

This work was supported by the National Key Research and Development Program of China (2018YFF0212902 and 2018YFB1308000), the National Natural Science Foundation of China (51805161), the Hunan Provincial Natural Science Foundation of China (2018JJ3187 and 2017JJ1015), the Guangdong Provincial Natural Science Foundation of China (2019A1515011961), the Research Project of Hunan Provincial Department of Education (19C0769), and the Changsha Science and Technology Program (No. kq1905019).

\section{References}

[1] N. V. Yetukuri and G. W. Fischer, "Planning the gmaw process by constraint propagation," Journal of Intelligent Manufacturing, vol. 8, no. 6, pp. 477-488, 1997.

[2] K. He, Q. Li, and J. Chen, "An arc stability evaluation approach for sw ac saw based on Lyapunov exponent of welding current," Measurement, vol. 46, no. 1, pp. 272-278, 2013.

[3] Y. Li, M. Xu, X. Liang, and W. Huang, "Application of bandwidth EMD and adaptive multiscale morphology analysis for incipient fault diagnosis of rolling bearings," IEEE Transactions on Industrial Electronics, vol. 64, no. 8, pp. 6506-6517, 2017.

[4] Y. Wang, Z. Wei, and J. Yang, "Feature trend extraction and adaptive density peaks search for intelligent fault diagnosis of machines," IEEE Transactions on Industrial Informatics, vol. 15, no. 1, pp. 105-115, 2019.
[5] S. Udit, T. Nikita, B. Gagarin et al., "Specific emitter identification based on variational mode decomposition and spectral features in single hop and relaying scenarios," IEEE Transactions on Information Forensics and Security, vol. 14, no. 3, pp. 581-591, 2019.

[6] F. Grimaccia, G. Gruosso, M. Mussetta, A. Niccolai, and R. E. Zich, "Design of tubular permanent magnet generators for vehicle energy harvesting by means of social network optimization," IEEE Transactions on Industrial Electronics, vol. 65, no. 2, pp. 1884-1892, 2018.

[7] M.-H. Lee, K.-K. Shyu, P.-L. Lee, C.-M. Huang, and Y.-J. Chiu, "Hardware implementation of emd using dsp and fpga for online signal processing," IEEE Transactions on Industrial Electronics, vol. 58, no. 6, pp. 2473-2481, 2011.

[8] N. E. Huang, Z. Shen, S. R. Long et al., “The empirical mode decomposition and the hilbert spectrum for nonlinear and non-stationary time series analysis," Proceedings of the Royal Society of London. Series A: Mathematical, Physical and Engineering Sciences, vol. 454, no. 1971, pp. 903-995, 1998.

[9] Y. Wang, R. Markert, J. Xiang, and W. Zheng, "Research on variational mode decomposition and its application in detecting rub-impact fault of the rotor system," Mechanical Systems and Signal Processing, vol. 60-61, pp. 243-251, 2015.

[10] K. Dragomiretskiy and D. Zosso, "Variational mode decomposition," IEEE Transactions on Signal Processing, vol. 62, no. 3, pp. 531-544, 2014.

[11] Y. Kai, W. Guofeng, D. Yi et al., "Early chatter identification based on an optimized variational mode decomposition," Mechanical Systems and Signal Processing, vol. 115, pp. 238254, 2019.

[12] M. Ali, A. Khan, and N. U. Rehman, "Hybrid multiscale wind speed forecasting based on variational mode decomposition," International Transactions on Electrical Energy Systems, vol. 28, no. 1, 2018.

[13] H. Bo, Y. H. Yang, Z. G. Luo et al., "Signal analysis of magnetic-control submerged arc welding seam tracking based on the variational mode decomposition," Electric Welding Machine, vol. 60-61, pp. 243-251, 2015.

[14] D. Camarena-Martinez, M. Valtierra-Rodriguez, C. A. PerezRamirez, J. P. Amezquita-Sanchez, R. de Jesus RomeroTroncoso, and A. Garcia-Perez, "Novel downsampling empirical mode decomposition approach for power quality analysis," IEEE Transactions on Industrial Electronics, vol. 63, no. 4, pp. 2369-2378, 2016.

[15] Z. Wu and N. E. Huang, "Ensemble empirical mode decomposition: a noise-assisted data analysis method," Advances in Adaptive Data Analysis, vol. 1, no. 1, pp. 1-41, 2009.

[16] J. Cheng, Y. Yang, and Y. Yang, "A rotating machinery fault diagnosis method based on local mean decomposition," Digital Signal Processing, vol. 22, no. 2, pp. 356-366, 2012.

[17] J. Wu, C. Wu, S. Cao, S. W. Or, C. Deng, and X. Shao, "Degradation data-driven time-to-failure prognostics approach for rolling element bearings in electrical machines," IEEE Transactions on Industrial Electronics, vol. 66, no. 1, pp. 529-539, 2019.

[18] K. T. Sweeney, S. F. McLoone, and T. E. Ward, "The use of ensemble empirical mode decomposition with canonical correlation analysis as a novel artifact removal technique," IEEE Transactions on Biomedical Engineering, vol. 60, no. 1, pp. 97-105, 2013.

[19] Z. Feng, M. Liang, Y. Zhang, and S. Hou, "Fault diagnosis for wind turbine planetary gearboxes via demodulation analysis based on ensemble empirical mode decomposition and energy separation," Renewable Energy, vol. 47, pp. 112-126, 2012. 
[20] R. F. De, R. Pintelon, J. Schoukens et al., "Reduction of the gibbs phenomenon applied on nonharmonic time base distortions," IEEE Transactions on Instrumentation and Measurement, vol. 54, no. 3, pp. 1118-1125, 2005.

[21] B. Alatas, "Acroa: artificial chemical reaction optimization algorithm for global optimization," Expert Systems with Applications, vol. 38, no. 10, pp. 13170-13180, 2011.

[22] Q. He, F. Kong, and R. Yan, "Subspace-based gearbox condition monitoring by kernel principal component analysis," Mechanical Systems and Signal Processing, vol. 21, no. 4, pp. 1755-1772, 2007.

[23] S. Wang, J. Lu, X. Gu, H. Du, and J. Yang, "Semi-supervised linear discriminant analysis for dimension reduction and classification," Pattern Recognition, vol. 57, pp. 179-189, 2016.

[24] Q. He, "Vibration signal classification by wavelet packet energy flow manifold learning," Journal of Sound and Vibration, vol. 332, no. 1, pp. 1881-1894, 2013.

[25] M. Belkin and P. Niyogi, "Laplacian eigenmaps for dimensionality reduction and data representation," Neural Computation, vol. 15, no. 6, pp. 1373-1396, 2003.

[26] X. He, S. Yan, Y. Hu, P. Niyogi, and H.-J. Zhang, "Face recognition using laplacian faces," IEEE Transactions on Pattern Analysis and Machine Intelligence, vol. 27, no. 3, pp. 328-340, 2005.

[27] X. Ding, Q. He, and N. Luo, "A fusion feature and its improvement based on locality preserving projections for rolling element bearing fault classification," Journal of Sound and Vibration, vol. 335, no. 1, pp. 367-383, 2015.

[28] J.-B. Yu, "Bearing performance degradation assessment using locality preserving projections," Expert Systems with Applications, vol. 38, no. 6, pp. 7440-7450, 2011.

[29] J. Yu, "Bearing performance degradation assessment using locality preserving projections and Gaussian mixture models," Mechanical Systems and Signal Processing, vol. 25, no. 7, pp. 2573-2588, 2011.

[30] Y. F. Peng, J. S. Cheng, and Y. Yang, "Adaptive sparsest narrow-band decomposition method and its applications to rolling element bearing fault diagnosis," Mechanical Systems and Signal Processing, vol. 85, no. 1, pp. 947-962, 2017. 